Introduction: In response to the Pulse Nightclub and Las Vegas mass shootings, staff from our Emergency Department (ED) at University Medical Center New Orleans designed a mass casualty incident (MCI) protocol aimed at preparing the entire hospital for high-volume, high-acuity incidents of unprecedented proportions. As we researched this effort, we discovered that no publically available framework currently exists to assist hospitals with creating their own comprehensive, functional MCI protocol.

Aim: To develop a framework to assist hospitals with creating MCI plans tailored to fit the needs of their individual facility.

Methods: Our hospital spent several years creating and refining an MCI protocol that is both comprehensive in addressing each service's needs and efficient for the staff expected to use it. Upon achieving the desired outcome of a well-functioning and tested protocol, the main contributors of the project met to create a consensus document on how we would approach the task with the benefit of hindsight.

Results: Our document is meant to serve as a framework for hospitals looking to build their own plan. It is not a template, but rather a guide on how to build an individualized plan that includes critical components that are key for success. It breaks the process down into manageable steps that are presented in an order that maximizes efficiency and includes important points to consider for each step. It encourages the user to tailor the protocol to their own unique needs.

Discussion: By sharing a framework based on our own best practices and lessons learned, we hope to make it easier for other hospitals to create MCI protocols and to open a dialogue with hospitals that have additional or differing opinions to share. Most importantly, we hope to inspire hospitals to work together as we race to prepare for worst-case scenarios of increasing magnitude.

Prehosp. Disaster Med. 2019;34(Suppl. 1):s90-s91

doi:10.1017/S1049023X19001882

Hemorrhage-control Tourniquets: How Intuitive are They? Ms. Nicole Ochs' ${ }^{1}$ Dr. Martha Sexton ${ }^{2}$, Mrs. Nicole McKenzie ${ }^{2}$, Dr. Paul Rega', Mr. Jonathon Ziehr ${ }^{3}$, Mr. Brent Parquette ${ }^{3}$, Dr. Brian Fink ${ }^{2}$

1. University of Mount Union, Alliance, United States

2. University Of Toledo, Toledo, United States

3. Lucas County Emergency Medical Service, Toledo, United States

Introduction: According to the Federal Bureau of Investigation (FBI), there were fifty active shooter incidents in 2016 and 2017. In the first five months of 2018, there have been 23 school shootings where someone was injured or killed. Hemorrhage-control tourniquets have proven their life-saving capability in the military and civilian EMS. Now, they are being advocated for use by civilians - the true "first responders." Since Combat Application Tourniquets (CATs) are strap-andwindlass devices, the question remains whether a naive population can intuitively apply them efficaciously.

Aim: To determine the efficacy (speed, correct placement) of a CAT by an interprofessional group of healthcare students naïve to tourniquet hemorrhage control.
Methods: Consenting students attempted to apply a CAT to a standardized patient with a simulated hemorrhaging brachial artery. No instruction was given except for the directions included in the package. Timing began upon removal of the tourniquet from the package and ended when the participants stated they completed their attempt. Errors in application were documented. Afterward, students received education and an opportunity to properly re-apply the tourniquet. The completion times of the students were compared to ten emergency medical technicians (EMT-P), serving as subject matter experts. Errors in application were categorized.

Results: 50 students from the following professions participated: Medicine, Nursing, Public Health, and Respiratory Therapy. The mean time of tourniquet application was 96.16 seconds (range: 25.12-226.31). This was statistically different from the EMT-Ps' time of 42.83 seconds (range: 23.89-82.94). Additionally, only five (10\%) placed the tourniquet correctly. Errors included improper location and windlass misuse or nonuse. The instructions were frequently critiqued for being difficult to read and containing confusing graphics.

Discussion: Provision of commercial tourniquets in public access areas must be accompanied by civilian education and the creation of CAT instructions that are simplistic, comprehensible, and suitably graphic.

Prehosp. Disaster Med. 2019;34(Suppl. 1):s91

doi:10.1017/S1049023X19001894

\section{Lessons from the Brussels Terrorist Attack}

Dr. Andreas Möbler

1. Emergency Department, CHU Brugmann, Brussels, Belgium

2. Medical Director of the Emergency Medical Response on March 22nd 2016, Brussels, Belgium

Introduction: On March 22, 2016, the capital of Europe was hit by two terrorist attacks. As terrorism becomes more and more violent, it is critical to learn and share experiences in order to enhance effectiveness in saving lives.

Methods: A field perspective and experience feedback from the Emergency Medical Response.

Results: The first attack hit the departure hall of the airport, which, due to its strategic role, relies upon a dedicated emergency plan. However, it focuses on airplane crashes and not on explosions in a crowded terminal. The second attack hit the subway at rush hour. An attack in such a confined environment is particularly challenging for the rescue teams, as injuries are worsened, access hindered, and exits numerous.

Eleven medical teams were sent in order to perform triage and provide vital care. The medical response was organized by two disaster response teams. Advanced Medical Posts were set up and the mass casualty plans of all hospitals were activated. Managing war injuries for civilian teams was challenging. Onsite care consisted essentially in prehospital damage control and burn care in order to ensure rapid evacuations for haemostatic surgery. 313 victims were dispatched to thirty hospitals. Another challenge was safety. Several threats were apparent and explosives were found on both sites. Lessons from Paris had prompted a review of our multiple sites Emergency Plan. One single way of communication was used and the evacuations 